\title{
Oxygen isotopic ratios in Martian water vapour observed by ACS MIR on board the ExoMars Trace Gas Orbiter
}

\author{
J. Alday ${ }^{1}$, C. F. Wilson ${ }^{1}$, P. G. J. Irwin ${ }^{1}$, K. S. Olsen ${ }^{2}$, L. Baggio ${ }^{2}$, F. Montmessin ${ }^{2}$, A. Trokhimovskiy ${ }^{3}$, \\ O. Korablev ${ }^{3}$, A. A. Fedorova ${ }^{3}$, D. A. Belyaev ${ }^{3}$, A. Grigoriev ${ }^{3}$, A. Patrakeev ${ }^{3}$, and A. Shakun \\ ${ }^{1}$ Department of Physics, University of Oxford, Oxford, UK \\ e-mail: juan.aldayparejo@physics.ox.ac.uk \\ ${ }^{2}$ LATMOS/CNRS, Paris, France \\ ${ }^{3}$ Space Research Institute (IKI), Moscow, Russia
}

Received 4 July 2019 / Accepted 25 August 2019

\begin{abstract}
Oxygen isotope ratios provide important constraints on the history of the Martian volatile system, revealing the impact of several processes that might fractionate them, such as atmospheric loss into space or interaction with the surface. We report infrared measurements of the Martian atmosphere obtained with the mid-infrared channel (MIR) of the Atmospheric Chemistry Suite (ACS), onboard the ExoMars Trace Gas Orbiter. Absorption lines of the three main oxygen isotopologues of water vapour $\left(\mathrm{H}_{2}^{16} \mathrm{O}, \mathrm{H}_{2}^{18} \mathrm{O}\right.$, and $\left.\mathrm{H}_{2}^{17} \mathrm{O}\right)$ observed in the transmission spectra allow, for the first time, the measurement of vertical profiles of the ${ }^{18} \mathrm{O} /{ }^{16} \mathrm{O}$ and ${ }^{17} \mathrm{O} /{ }^{16} \mathrm{O}$ ratios in atmospheric water vapour. The observed ratios are enriched with respect to Earth-like values $\left(\delta^{18} \mathrm{O}=200 \pm 80 \%\right.$ and $\delta^{17} \mathrm{O}=230 \pm 110 \%$ corresponding to the Vienna Standard Mean Ocean Water). The vertical structure of these ratios does not appear to show significant evidence of altitudinal variations.
\end{abstract}

Key words. planets and satellites: atmospheres - planets and satellites: composition - planets and satellites: terrestrial planets radiative transfer - infrared: planetary systems

\section{Introduction}

Enrichment of isotopic ratios in the Martian atmosphere with respect to the Earth in species such as $\mathrm{H}, \mathrm{N}$, or noble gases has often been understood as evidence of atmospheric escape into space. In particular, the enrichment of the $\mathrm{D} / \mathrm{H}$ ratio in water vapour of approximately a factor of six, as compared to the terrestrial ratio (Owen et al. 1988), is believed to be indirect evidence of the wetter and denser atmosphere that Mars may have once harboured. Since the first detections of the Martian $\mathrm{D} / \mathrm{H}$ ratio, several remote observations and in situ measurements have proved its strong seasonal and regional variability (Webster et al. 2013; Villanueva et al. 2015; Aoki et al. 2015; Krasnopolsky 2015; Encrenaz et al. 2016; Vandaele et al. 2019), mainly due to the isotopic fractionation during the condensation and sublimation of the polar caps (Montmessin et al. 2005).

In contrast to the large enrichment in the heavy isotope measured in the $\mathrm{D} / \mathrm{H}$ ratio, the isotope ratios measured in oxygen $\left({ }^{18} \mathrm{O} /{ }^{16} \mathrm{O}\right.$ and $\left.{ }^{17} \mathrm{O} /{ }^{16} \mathrm{O}\right)$ appear to be more consistent with telluric values. This has been interpreted to be due to the presence of a large reservoir that mitigates the effect of escape of oxygen atoms into space (Jakosky 1997).

The only peer-reviewed measurement of the ${ }^{18} \mathrm{O} /{ }^{16} \mathrm{O}$ in water vapour was reported by Webster et al. (2013) using the Sample Analysis at Mars (SAM) on the Curiosity rover, showing an enrichment of $\delta^{18} \mathrm{O}=84 \pm 10 \%$ with respect to the Vienna Standard Mean Ocean Water (VSMOW). Oxygen isotope ratios are usually given as the deviation of the measured ratio $(R)$ with respect to the VSMOW $\left(R_{\mathrm{S}}\right)$ in units of per mil $(\%)$ (e.g. $\left.\delta^{18} \mathrm{O}=\left(R / R_{\mathrm{s}}-1\right) \times 1000\right)$. The standard ratios, as defined by VSMOW, are given by ${ }^{18} \mathrm{O} /{ }^{16} \mathrm{O}=2005.2 \times 10^{-6}$ and ${ }^{17} \mathrm{O} /{ }^{16} \mathrm{O}=379.9 \times 10^{-6}$. In a conference abstract, Bjoraker et al. (1989) reported depletion of the heavier isotopes $\left(\delta^{18} \mathrm{O}=\right.$ $-100 \pm 30 \%$ and $\delta^{17} \mathrm{O}=-50 \pm 10 \%$ ) using observations made with the Fourier Transform Spectrometer on the Kuiper Airborne Observatory.

Atmospheric losses from $\mathrm{H}$ and $\mathrm{O}$ are expected to be linked as are the isotopic ratios $\mathrm{D} / \mathrm{H}$ and ${ }^{18} \mathrm{O} /{ }^{16} \mathrm{O}$. Nevertheless, unlike hydrogen, whose main source comes from the Martian water reservoir, the oxygen atoms in water vapour are expected to interact with those from the $\mathrm{CO}_{2}$ reservoir and minerals in the crust; hence the connection is likely to be complex (Jakosky \& Phillips 2001). Early ground-based measurements of the oxygen isotopic composition of $\mathrm{CO}_{2}$ showed a depletion of ${ }^{18} \mathrm{O}$ with respect to ${ }^{16} \mathrm{O}\left(\delta^{18} \mathrm{O}=-40 \pm 130 \%\right.$, Schrey et al. 1986; $\delta^{18} \mathrm{O}=-130 \pm 80 \%$, Krasnopolsky et al. 1996), but more recent observations showed an enrichment consistent with telluric values $\left(\delta^{18} \mathrm{O}=18 \pm 18 \%\right.$, Krasnopolsky et al. 2007). The oxygen isotopic ratio has also been determined using in situ measurements by different rovers and landers. Measurements using the Neutral Mass Spectrometer on the Viking landers reported the $\delta^{18} \mathrm{O}$ in atmospheric $\mathrm{CO}_{2}$ to be consistent with terrestrial values to within 5\% (Nier \& McElroy 1977). Niles et al. (2010) report measurements made by the Phoenix lander of $\delta^{18} \mathrm{O}=31 \pm 5.7 \%$ and Webster et al. (2013) report values for both the ${ }^{18} \mathrm{O}$ and ${ }^{17} \mathrm{O}$ ratios in $\mathrm{CO}_{2}\left(\delta^{18} \mathrm{O}=48 \pm 5 \% ; \delta^{17} \mathrm{O}=24 \pm 5 \% 0\right)$, which is consistent with the expectations from mass-dependent fractionation.

In addition to the evolution of the Martian volatile system throughout history that is responsible for the isotopic composition of the bulk atmosphere, there are several fractionation 
processes which may give rise to spatial and seasonal variations in the present-day atmosphere. Condensation of $\mathrm{CO}_{2}$ and $\mathrm{H}_{2} \mathrm{O}$ into ice preferentially leaves the ${ }^{16} \mathrm{O}$ in the atmosphere, producing a depletion of $\delta^{18} \mathrm{O}$. This process might give rise to vertical fractionation, if ice clouds are formed, and to a seasonal pattern due to the sublimation and condensation of the polar caps (Montmessin et al. 2005). In addition, a daily fractionation pattern can occur during the gas adsorption and desorption on the Martian regolith, presumably following similar trends as predicted for the $\mathrm{D} / \mathrm{H}$ ratio by $\mathrm{Hu}$ (2019), but with a different magnitude.

In this study, oxygen isotopic ratios in water vapour are measured using solar occultation observations made by the Atmospheric Chemistry Suite (ACS) onboard ExoMars Trace Gas Orbiter (Korablev et al. 2017). These measurements allow, for the first time, the determination of vertical profiles for the ratios, potentially enabling the determination of altitudedependant fractionation processes. The ACS dataset also allows the measurement of the ratios in different locations and seasons, helping to get insight into the several processes that produce the fractionation.

\section{ACS instrument and measurements}

The Atmospheric Chemistry Suite (ACS) consists of a set of three infrared spectrometers designed to establish a detailed inventory of the trace gases in the martian atmosphere: a spectrometer combining an acousto-optic tunable filter (AOTF) with an echelle grating in the near infrared (NIR), an echelle cross-dispersion spectrometer in the middle infrared (MIR), and a Fourier Transform spectrometer for the thermal infrared (TIRVIM), covering a total wavelength range from 0.7 to $17 \mu \mathrm{m}$ (Korablev et al. 2017).

The MIR channel, used in this study, is an echelle crossdispersion spectrometer dedicated to solar occultation measurements in the $2.3-4.2 \mu \mathrm{m}$ range. It is a new concept designed for obtaining high spectral resolution $(\lambda / \Delta \lambda \approx 30000-50000)$ in a wide instantaneous spectral width $(0.15-0.3 \mu \mathrm{m})$, allowing the simultaneous detection and mapping of several atmospheric species. To cover the full spectral coverage, ACS MIR incorporates a secondary movable grating, which allows the projection of 7-17 diffraction orders on to a $512 \times 640$ pixel detector, depending on the tilt of the secondary grating (Korablev et al. 2017).

In this work, we use ACS MIR data obtained using the secondary grating position 5 . In this setup, each acquired detector frame contains 12 adjacent diffraction orders (226-237), covering a spectral range from 2.57 to $2.64 \mu \mathrm{m}$. Several spectral windows are then selected from diffraction orders 226-231, which contain absorption lines of the three main oxygen isotopologues in water vapour $\left(\mathrm{H}_{2}^{16} \mathrm{O}, \mathrm{H}_{2}^{18} \mathrm{O}, \mathrm{H}_{2}^{17} \mathrm{O}\right)$, and $\mathrm{CO}_{2}$ (see Fig. 1). Isotopic signatures in $\mathrm{CO}_{2}$ can also be measured using this secondary grating position, but the profiling of these species is beyond the scope of this work, and will be analysed in the future.

ACS MIR solar occultations observations are especially suitable for the measurement of trace gases due to the great brightness of the Sun, which results in high signal to noise $(\mathrm{S} / \mathrm{N})$ spectra, and the long observed path length, which results in an observed air mass about 10 times greater than when observing the nadir. ACS MIR observes the Sun from 0 to $200 \mathrm{~km}$ above the martian surface within 3-6 min. In each individual acquisition, 200 consecutive frames are stacked together on-board. The integration time of a single frame for secondary grating position
5 is $3 \mathrm{~ms}$, but each of the 200 readouts from the detector takes approximately $10 \mathrm{~ms}$, with one acquisition lasting $2.1 \mathrm{~s}$.

In each occultation, the uppermost heights are averaged to obtain a reference solar spectrum, which is then used to generate the transmission spectra. On the other hand, a dark signal, combination of the detector dark current and the surrounding thermal background emission, is estimated from the lowermost observations, where the Sun is completely concealed by the Martian surface, and refined in dedicated observations of open space. The spectral calibration of the measurement is performed using solar lines and comparing them to the ACE-FTS solar atlas (Hase et al. 2010). Then, it is further refined using observed atmospheric absorption lines of $\mathrm{CO}_{2}$ and $\mathrm{H}_{2} \mathrm{O}$.

The light projected on the detector frame appears to produce a double image, with one of the images being slightly shifted with respect to the other and having lower intensity. The cause of this doubled-image is still unknown and needs to be tracked down, although it is presumably the result of a partial fracture in one of the lenses. Light coming from part of the slit is unaffected by this optical effect. Nevertheless, this portion of the slit is less bright, and we choose to optimise the photometry in the portion where the doubling is present. Further detail about this effect will be provided in future publications by the ACS team. In the present work the spectra are fitted using an instrument line shape (ILS) consisting of a superposition of two Gaussian functions in order to model the doubling of the absorption lines (see Fig. 2). The relative depth of the two Gaussians varies depending on the selected row on the detector frame, with wavenumber, and in different observations. For each occultation and spectral window, the parameters describing the ILS are fitted using the following assumptions. First of all, the full width at half maximum (FWHM) of the two Gaussians is the same, and is constant in wavelength units. Secondly, the relative offset of the centre of the two Gaussians does not vary with wavenumber. In the third place, the relative depth of the two Gaussians is linearly varying with wavenumber. Lastly, the same parameters apply to all acquisitions during one solar occultation.

Typical values of the measured spectral resolution range from approximately $0.12-0.15 \mathrm{~cm}^{-1}$. The offset of the second Gaussian is shifted typically $0.16 \mathrm{~cm}^{-1}$ from the centre of the main Gaussian, and its strength does not typically exceed $25 \%$ of that of the main one (see Fig. 2).

In this work, we focus on eight ACS MIR observations obtained during the first three months of operations to measure the vertical structure of the oxygen isotopic ratios in water vapour. These occultations are chosen due to the strong oxygen isotopic absorption signature they show, allowing a careful validation of the results. Table 1 shows the orbital characteristics of the observations used in this study.

\section{Analysis of the observations}

In this work, the NEMESIS retrieval algorithm (Irwin et al. 2008), which works under the optimal estimation formalism (Rodgers 2000), is applied to retrieve the atmospheric state from the ACS MIR spectra. In particular, simultaneous vertical profiles of pressure, temperature, and volume mixing ratio for the three main oxygen isotopologues of water vapour are obtained for each observation, using the spectral windows shown in Fig. 1.

Pressure and temperature profiles can be constrained under the assumption of hydrostatic equilibrium and a known $\mathrm{CO}_{2}$ volume mixing ratio profile, which in this case is obtained from the Mars Climate Database (Forget et al. 1999). This technique was applied in stellar occultation observations using SPICAM 
J. Alday et al.: Oxygen isotopic ratios in Martian water vapour observed by ACS MIR on board the ExoMars Trace Gas Orbiter
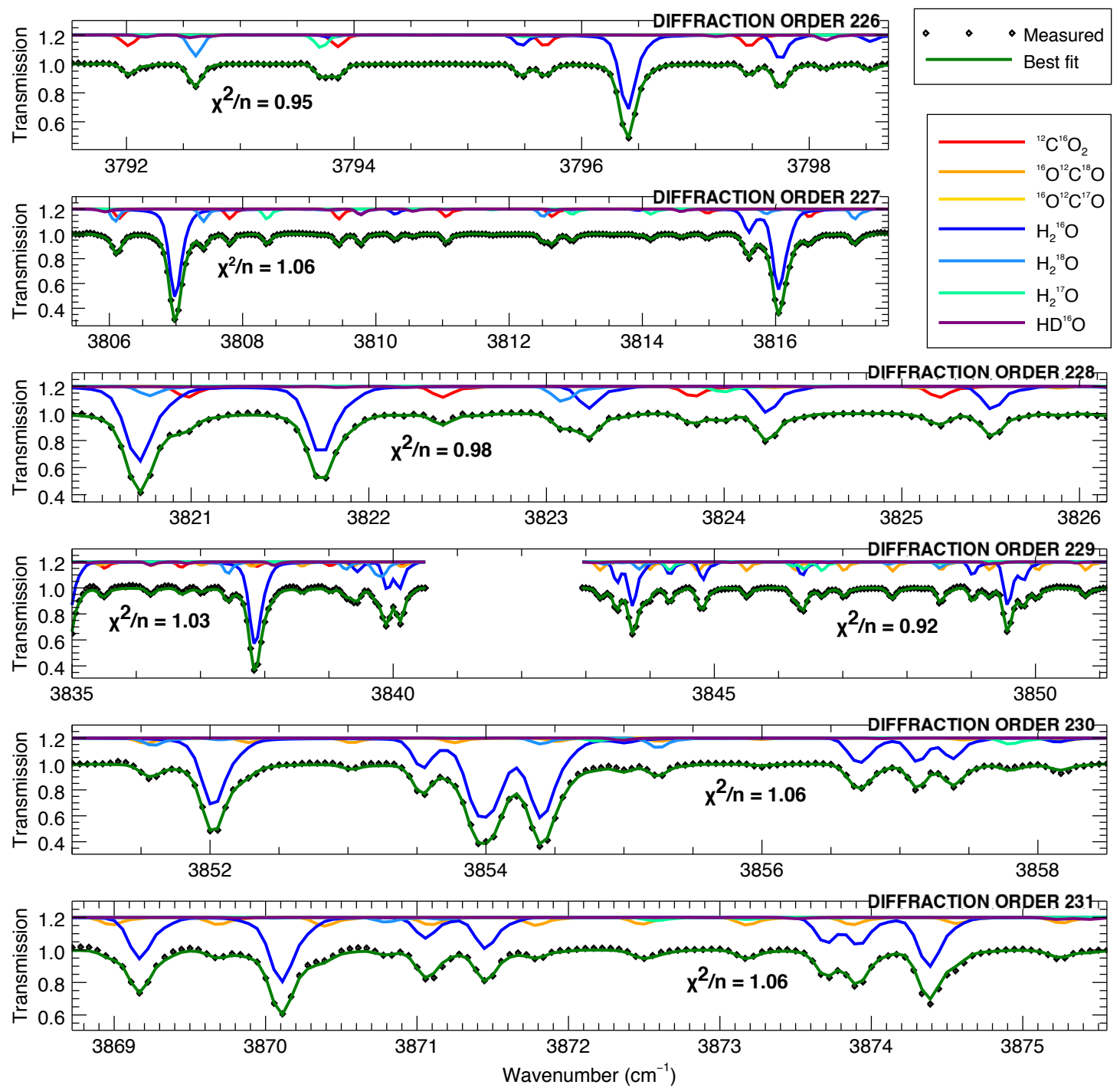

Fig. 1. ACS MIR normalised transmission spectra obtained at tangent height of $16.8 \mathrm{~km}$ above martian ellipsoid in observation made in orbit 2233 , using spectral windows from diffraction orders 226-231. The green line is the best fit to the data, while the coloured lines represent the contribution to the absorption from each gas. The contribution from each gas is shifted 0.2 for the clarity of the figure.
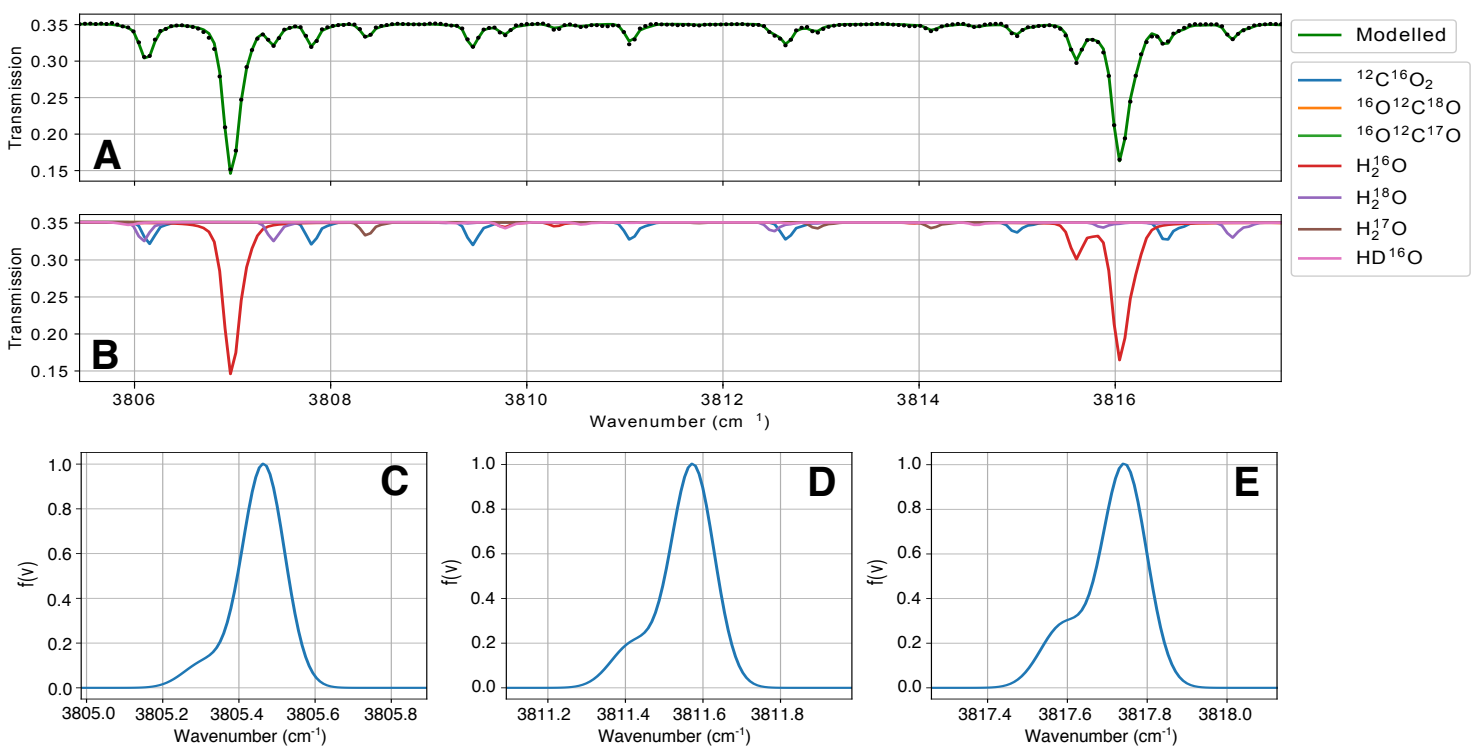

Fig. 2. ACS MIR transmission spectrum for diffraction order 227 obtained at tangent height of $11.4 \mathrm{~km}$ above the martian ellipsoid in the observation made in orbit 2520. (A) Measurement and best fit to the data. (B) Contribution from each gas to the spectrum. (C), (D) and (E) show the ILS function used at the first, middle, and last spectral point of the spectrum. 
Table 1. Orbital parameters for ACS MIR observations selected for this analysis.

\begin{tabular}{lcccccc}
\hline \hline Orbit & Ingress/Egress & Date (UTC) & Latitude $\left(^{\circ}\right)$ & Longitude $\left(^{\circ}\right)$ & Ls $\left(^{\circ}\right)$ & Local time \\
\hline 1917 & Ingress & $2018-04-29$ & -52 & -139 & 167 & 17 \\
1924 & Ingress & $2018-04-29$ & -50 & 21 & 167 & 18 \\
1961 & Ingress & $2018-05-02$ & -37 & 42 & 169 & 18 \\
2233 & Egress & $2018-05-24$ & -25 & -33 & 181 & 6 \\
2502 & Ingress & $2018-06-15$ & 62 & -11 & 194 & 17 \\
2520 & Ingress & $2018-06-17$ & 59 & -167 & 195 & 17 \\
2929 & Egress & $2018-07-20$ & 53 & -157 & 215 & 7 \\
2984 & Egress & $2018-07-25$ & 61 & 75 & 218 & 8 \\
\hline
\end{tabular}

on Mars Express (Quémerais et al. 2006; Montmessin et al. 2006; Forget et al. 2009). In those analyses, density profiles are obtained from the $\mathrm{CO}_{2}$ absorption, and temperature was then derived assuming hydrostatic equilibrium and the ideal gas law. In this work the same assumptions are made, but retrieving a vertical profile of temperature $T(z)$ and fixing the surface pressure $p\left(z_{0}\right)$ as given by the Mars Climate Database (MCD). Then, the rest of the pressure levels are re-computed based on the hydrostatic equilibrium equation given by

$p(z)=p\left(z_{0}\right) \cdot \exp \left(-\int_{z_{0}}^{z} \frac{g(\bar{z}) M(\bar{z})}{T(\bar{z}) k_{\mathrm{B}}} \mathrm{d} \bar{z}\right)$,

where $g(z)$ is the gravity, $M(z)$ is the molecular weight, which is taken from the $\mathrm{MCD}$, and $k_{\mathrm{B}}$ is the Boltzmann constant $\left(k_{\mathrm{B}}=1.38 \times 10^{-23} \mathrm{~J} \mathrm{~K}^{-1}\right)$. This scheme provides the advantage of updating the temperature levels in each iteration, and therefore updating the temperature dependence of the absorption lines.

The vertical profiles of the different water vapour isotopologues are retrieved from the absorption lines by each gas (see Fig. 1). All gaseous absorption is modelled using pre-computed look-up tables using line-by-line modelling. The $\mathrm{H}_{2} \mathrm{O}$ absorption lines are modelled using the absorption coefficients for a $\mathrm{CO}_{2}$-dominated atmosphere published by Gamache et al. (2016), and later updated with laboratory measurements by Devi et al. (2017) for HDO. Previous studies on Mars have often applied a constant scaling of 1.67 with respect to the air-broadened coefficients in an Earth-like atmosphere $\left(\mathrm{N}_{2}+\mathrm{O}_{2}\right)$ derived by Brown et al. (2007) to simulate the effect of a $\mathrm{CO}_{2}$-dominated atmosphere. Nevertheless, Brown et al. (2007) point out that the measured ratio of $\mathrm{CO}_{2}$ to air broadened coefficients shows large variability around the mean value of 1.67 (0.95-3.07), and therefore the "simple scaling of the coefficients for $\mathrm{N}_{2}$ broadening does not provide a reliable set for $\mathrm{CO}_{2}$ broadening of water" (Brown et al. 2007). Gamache et al. (2016) published a spectral line list for the water isotopologues, which simulates the line shape parameters using the Modified Complex Robert-Bonamy formalism (MCRB), which in general yields to average uncertainties of $\approx 5 \%$ and should provide a more accurate representation than a constant scaling factor. Therefore, we use the spectral line list of Gamache et al. (2016) to model the gaseous absorption of the water isotopologues. In the case of $\mathrm{CO}_{2}$, it is the 2016 edition of the HITRAN spectroscopic line list (Gordon et al. 2017) which is used (Olsen et al. 2019).

The optimal estimation formalism works on the basis of finding a solution that replicates the measured spectrum with a solution that is closest to an a priori state of the atmosphere. The a priori state vector is accompanied with its covariance matrix $\mathbf{S}_{a}$, which represents the uncertainty in the system prior to the retrieval. The retrieved uncertainties are expressed on the basis of this matrix, given by

$\mathbf{S}_{t}=\left(\mathbf{K}^{T} \mathbf{S}_{\epsilon}^{-1} \mathbf{K}+\mathbf{S}_{x}^{-1}\right)^{-1}$,

where $\mathbf{S}_{t}$ is the retrieved covariance matrix, $\mathbf{S}_{\epsilon}$ is the measurement covariance matrix, representing the uncertainty in the spectra, and $\mathbf{K}$ is the Jacobian matrix (i.e. the rate of change of the transmission spectra with respect to each element in the state vector). The noise in the transmission spectra is adjusted, after a first retrieval, to be consistent with the magnitude of the observed residuals (the difference between the observed and modelled spectra). Then, the retrieval is repeated using this adjusted measurement uncertainty.

In the case in which there is no information contained in the spectra for some element of the state vector (i.e. $\mathbf{K}^{T} \mathbf{S}_{\epsilon}^{-1} \mathbf{K}=0$ ), then the retrieved uncertainty would be equal to the a priori. This approach might be misleading in some cases. If, for example, one specifies a $20 \%$ uncertainty in the a priori profile, then the retrieved profile will have an uncertainty of $20 \%$ even at altitudes where the observation provides no constraint. One solution would be to increase the uncertainties in the a priori. However, when not enough constraint is applied in the a priori, the retrieval might get ill-conditioned, leading to highly unrealistic vertical oscillations in the retrieved profiles (Irwin et al. 2008).

In order to mitigate the effect of the a priori uncertainty in the retrieval errors, we introduce a correcting factor. This factor, applied before by Irwin et al. (2019), is given by

$\sigma(i)=\frac{1}{\sqrt{\frac{1}{\mathbf{S}_{t}(i, i)}-\frac{1}{\mathbf{S}_{a}(i, i)}}}$,

where $\sigma(i)$ is the corrected uncertainty of the $i$ th element of the state vector. On the basis of retrievals on synthetic spectra, this approach is found to provide a more reliable estimation of the uncertainties (i.e. those that reflect the true uncertainty with respect to the state vector used to generate the synthetic spectra), and provides a reliable representation of where the retrieval is sensitive to the observation.

This retrieval scheme, including the adjustment of the parameters defining the ILS (see Sect. 2), is applied independently to each spectral window. However, not all atmospheric parameters can be determined from every spectral window. In particular, pressure and temperature profiles are obtained using the absorption lines of ${ }^{12} \mathrm{C}^{16} \mathrm{O}_{2}$ in diffraction orders 226, 227 and 228. Once determined, the weighted-averaged retrieved pressure and temperature profiles are fixed, and retrievals for the water vapour isotopologues are independently performed in the remaining spectral windows.

In order to combine the retrieved profiles from each spectral window, we calculate the weighted average of the gas profiles, 

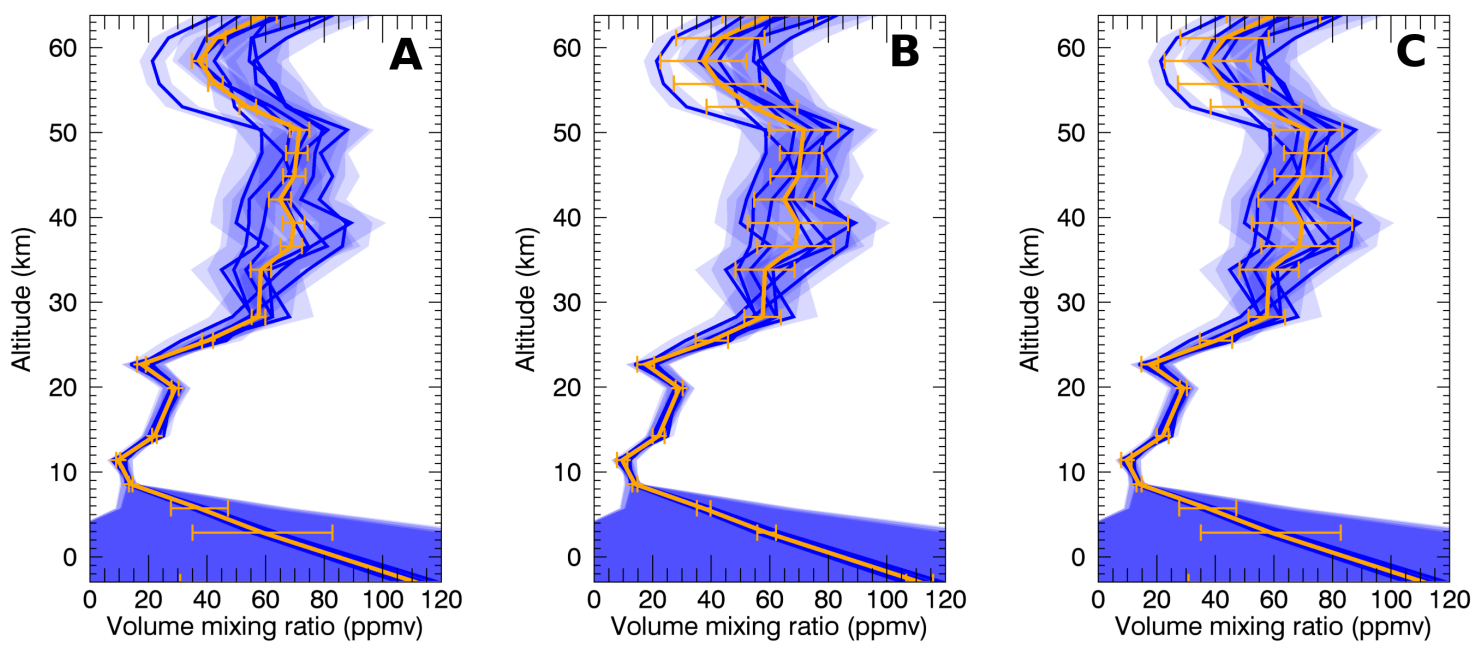

Fig. 3. Retrieved $\mathrm{H}_{2}^{16} \mathrm{O}$ vertical profiles from all spectral windows (blue lines) in the observation performed during orbit 2520 , as well as the weighted-averaged profile (orange line). Uncertainties in the averaged profile in panel $A$ are derived from the propagation of errors from each retrieved profile. Uncertainties in panel $B$ are calculated as the weighted standard deviation from all the profiles. Panel $C$ shows the combination of the two previous panels, selecting the larger of the two methods. Altitudes are defined with respect to the Martian ellipsoid.

using weights given by the inverse of the variance at each altitude $\left(\sigma(z)^{-2}\right)$. Figure 3 shows an example of the retrieved $\mathrm{H}_{2}^{16} \mathrm{O}$ volume mixing ratio vertical profiles (orbit 2520, see Table 1) in each spectral window, as well as the averaged profile.

Standard error propagation of the retrieved uncertainties in the averaged vertical profile (see panel A in Fig. 3) does not appear to reflect the true uncertainty of the retrieval, as it is smaller than the variation of the retrieved profiles in each spectral window. In contrast, the uncertainty shown in panel B is calculated as the weighted standard deviation of all profiles. This approach appears to give a better representation of the retrieved uncertainty. Nevertheless, it underestimates the uncertainties in regions where there is no information in the spectra, as they all tend to the a priori state. Errorbars shown in panel $\mathrm{C}$ show the combination of the two previous approaches, selecting the one that provides a higher value. We consider this method to provide a more accurate representation of the true uncertainty, reflecting the variation of the several spectral windows, as well as the lack of information in the spectra.

This approach is applied to all retrieved profiles $\left(\mathrm{H}_{2}^{16} \mathrm{O}\right.$, $\mathrm{H}_{2}^{18} \mathrm{O}, \mathrm{H}_{2}^{17} \mathrm{O}$, pressure and temperature). Figure 4 shows the vertical profiles derived from the observations in orbits 1924 and 2929 (see Table 1). Retrieved profiles appear to be consistent as derived from the several spectral windows, showing the same altitude trends.

Determination of the pressure and temperature profiles is essential for the accurate derivation of the isotope ratios, which requires a good representation of the temperature field, as temperature impacts the absorption line strength and width differently for each water isotopologue. In order to validate the retrieved atmospheric structure, the results are compared to those retrieved from the NIR channel on ACS, which performs simultaneous measurements with the MIR channel. The NIR profiles are measured exploiting the temperature dependance of the $\mathrm{CO}_{2}$ rotational band at $1.57 \mu \mathrm{m}$ (Fedorova et al. 2019), which is a different method than the one applied in this work, which exploits the assumption of hydrostatic equilibrium. In addition, we search for collocated observations made with the Mars Climate Sounder (MCS) onboard Mars Reconnaissance Orbiter (MRO), applying criteria on location $\left( \pm 1^{\circ}\right.$ latitude and longitude), local time $( \pm 2 \mathrm{~h})$, and solar longitude $\left( \pm 0.5^{\circ}\right)$. We find one MCS observation (Latitude $=64^{\circ}$; Longitude $=12^{\circ}$;
$L_{\mathrm{s}}=194^{\circ}$; Local time $=15 \mathrm{~h}$ ) fulfilling the criteria as compared to ACS MIR observation made in orbit 2502 (see Table 1). Figure 5 shows the comparison between the NIR, MIR and MCS pressure-temperature profiles, exhibiting a good agreement between the different datasets, and increasing the confidence of the measurements and methods.

The volume mixing ratio profiles for the different isotopologues allow the derivation of the oxygen isotope ratios, which are calculated using $R\left({ }^{x} \mathrm{O}\right)=\left[\mathrm{H}_{2}^{x} \mathrm{O}\right] /\left[\mathrm{H}_{2}^{16} \mathrm{O}\right]$, where ${ }^{x} \mathrm{O}$ stands for ${ }^{18} \mathrm{O}$ and ${ }^{17} \mathrm{O}$. The deviation with respect to VSMOW is then calculated using $\delta^{x} \mathrm{O}=\left(R\left({ }^{x} \mathrm{O}\right) / R_{\mathrm{S}}\left({ }^{x} \mathrm{O}\right)-1\right) \times 1000$, where $R_{\mathrm{s}}$ represents the standard isotope ratios $\left(R_{\mathrm{s}}\left({ }^{18} \mathrm{O}\right)=2005.2 \times 10^{-6}\right.$ and $\left.R_{\mathrm{s}}\left({ }^{17} \mathrm{O}\right)=379.9 \times 10^{-6}\right)$. Figure 6 shows the retrieved profiles for all observations in Table 1, as well as the resulting isotopic ratios. The derived profiles for the oxygen isotopes in water vapour show a general enrichment in heavier isotopes with respect to telluric values.

In order to reduce the retrieved uncertainties and get an insight of the overall trends of the ratios, we express all vertical profiles in the same altitude grid, and calculate the weighted average over all analysed observations, as shown in Fig. 7. Both the ${ }^{18} \mathrm{O} /{ }^{16} \mathrm{O}$ and ${ }^{17} \mathrm{O} /{ }^{16} \mathrm{O}$ ratios appear to be relatively constant in the altitude range where ACS MIR is most sensitive. The ${ }^{18} \mathrm{O} /{ }^{16} \mathrm{O}$ ratio might show a slight enrichment with altitude, but this is within the order of the uncertainties. Assuming the isotope ratios are well mixed, it is possible to calculate an average value for all altitudes, which is $\delta^{18} \mathrm{O}=200 \pm 80 \%$ and $\delta^{17} \mathrm{O}=230 \pm 110 \%$, where the uncertainties correspond to the ones derived at the most constrained altitude.

\section{Discussion}

Transmission spectra acquired by the Atmospheric Chemistry Suite on the ExoMars Trace Gas Orbiter reveal several absorption lines of the three main oxygen isotopologues in water vapour $\left(\mathrm{H}_{2}^{16} \mathrm{O}, \mathrm{H}_{2}^{18} \mathrm{O}\right.$ and $\left.\mathrm{H}_{2}^{17} \mathrm{O}\right)$, between 2.57 and $2.64 \mu \mathrm{m}$. Eight solar occultation observations are analysed in this work sampling different latitudes and longitudes, when Mars was within a range of solar longitudes of $167^{\circ}$ and $218^{\circ}$.

Atmospheric retrievals using the NEMESIS radiative transfer code allow the determination of the vertical profiles of the oxygen isotope ratios in $\mathrm{H}_{2} \mathrm{O}$ for the first time. Despite the rather 

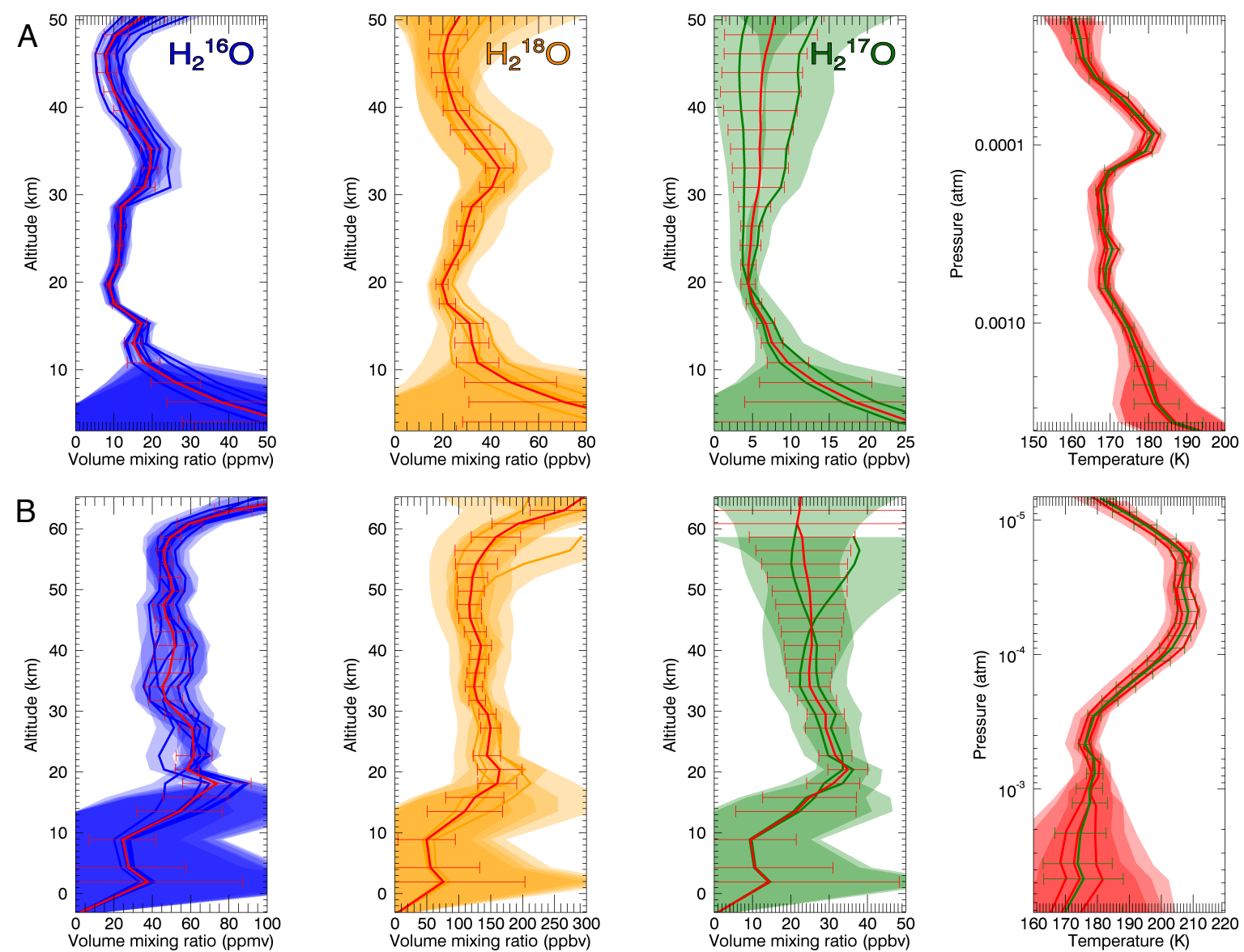

Fig. 4. Retrieved vertical profiles of temperature and volume mixing ratios of $\mathrm{H}_{2}^{16} \mathrm{O}, \mathrm{H}_{2}^{18} \mathrm{O}$ and $\mathrm{H}_{2}^{17} \mathrm{O}$, for the observations made in orbits $1924(A)$ and $2929(B)$. The retrieved profiles from several spectral windows are shown, as well as the derived averaged profile. Altitudes are defined with respect to the Martian ellipsoid.
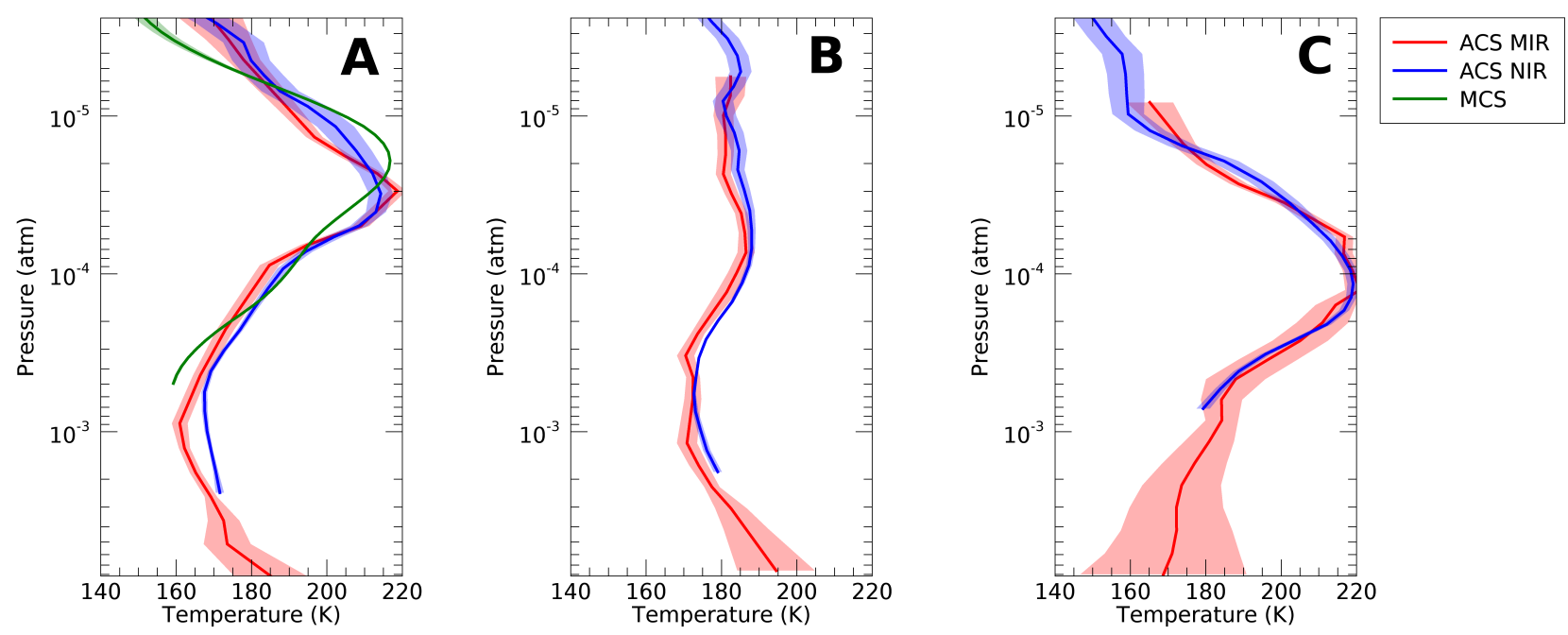

Fig. 5. Retrieved temperature and pressure profiles measured by the MIR and NIR channels in the solar occultation observations performed in orbits $2502(A), 2520(B)$ and $2984(C)$. Panel A: retrieved temperature and pressure profile from one MCS observation made in similar conditions as in ACS orbit 2502 (see text).

different conditions found in the atmosphere in terms of pressure, temperature and atmospheric water content in these eight observations, the retrieved profiles for the oxygen ratios $\left({ }^{18} \mathrm{O} /{ }^{16} \mathrm{O}\right.$ and ${ }^{17} \mathrm{O} /{ }^{16} \mathrm{O}$ ) do not appear to show differences greater than the level of the uncertainties.

Spatial, seasonal and vertical variations of the $\mathrm{O}$ isotopic ratios in water vapour due to evaporation and condensation processes are expected to follow similar trends as predicted for the $\mathrm{D} / \mathrm{H}$ ratio. Montmessin et al. (2005) predicts seasonal variations of the $\mathrm{D} / \mathrm{H}$ ratio of a factor of approximately 2.5 , corresponding to the sublimation and condensation of the polar caps, and Villanueva et al. (2015) measured variations of approximately a factor of 3 in different locations. Merlivat \& Nief (1967) and Majoube (1970) provide empirical formulas for the 

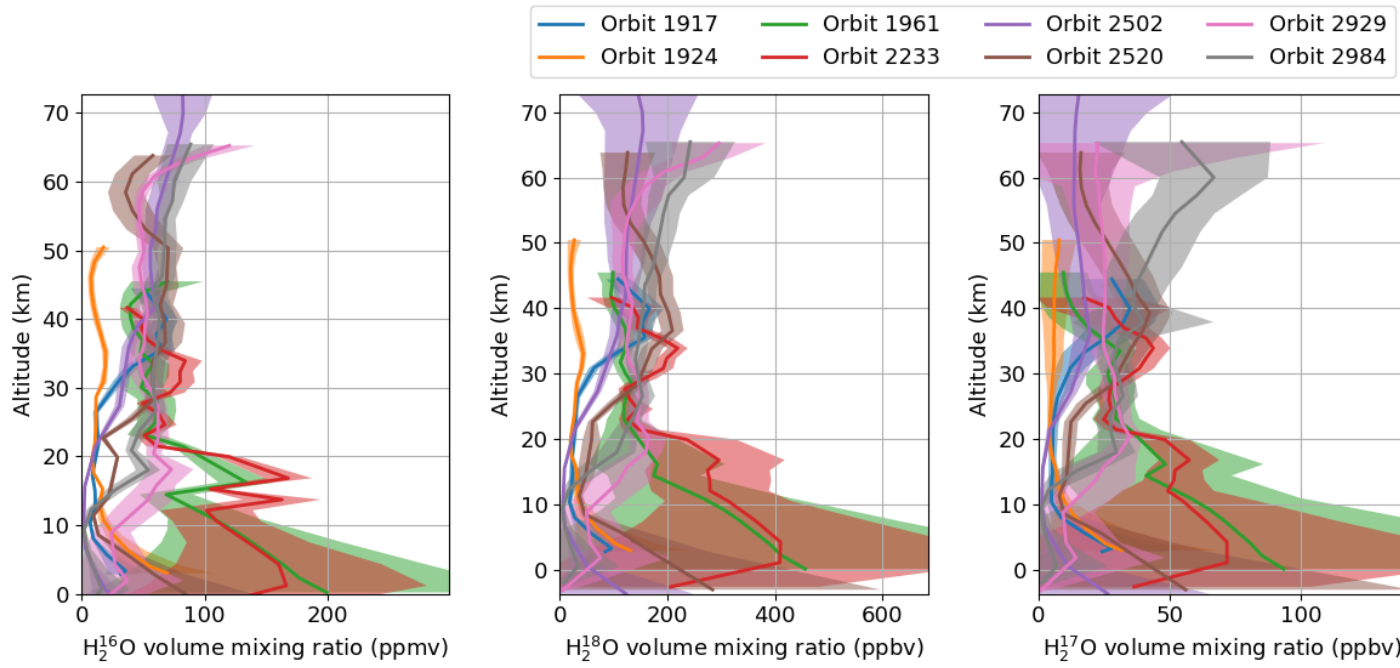

$\mathrm{H}_{2}^{16} \mathrm{O}$ volume mixing ratio (ppmv)
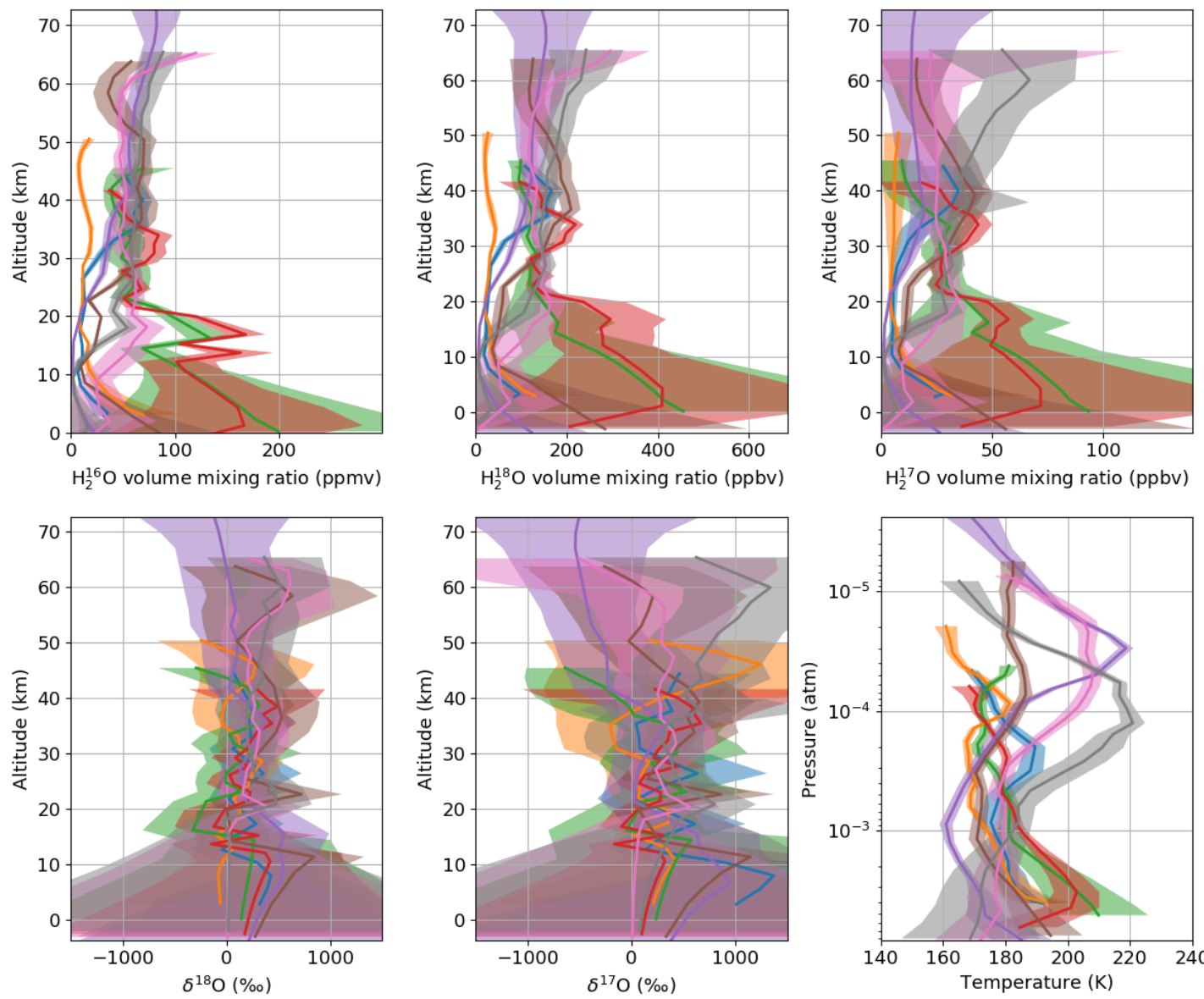

$\mathrm{H}_{2}^{17} \mathrm{O}$ volume mixing ratio (ppbv)

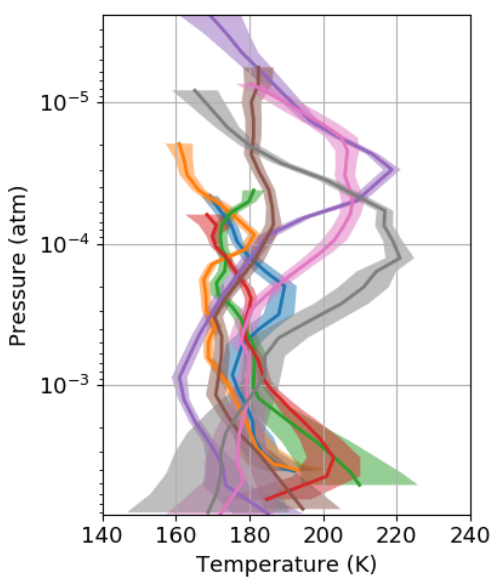

Fig. 6. Retrieved vertical profiles obtained for each of the processed observations. The isotopic ratios are expressed as deviations with respect to the VSMOW, which represents Earth-like isotope ratios. Altitudes are defined with respect to the Martian ellipsoid.

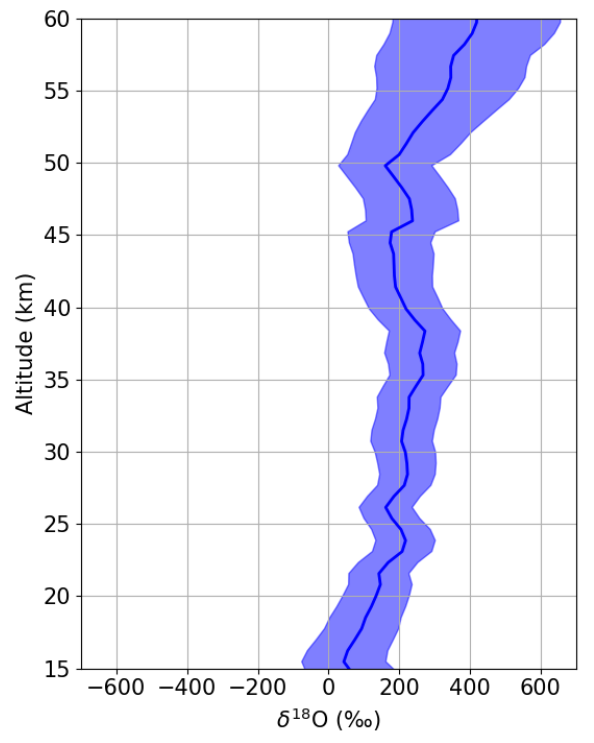

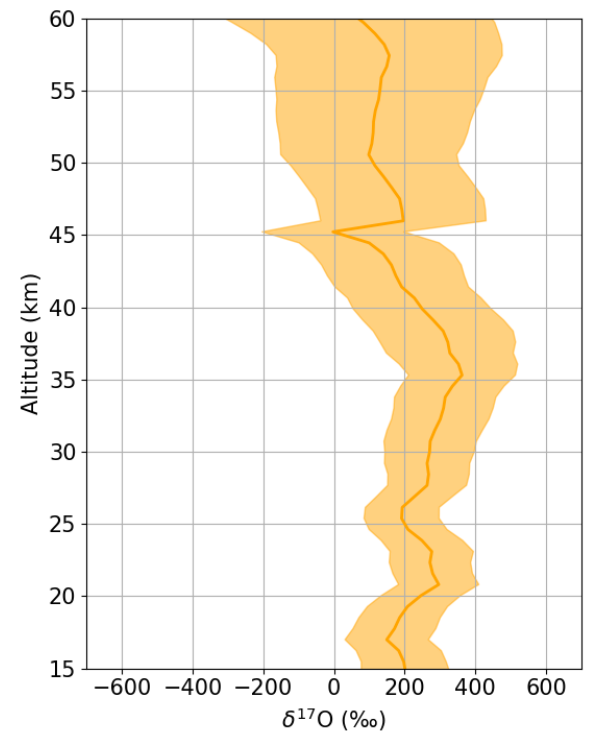

Fig. 7. Weighted-averaged vertical profiles of the oxygen isotopic ratios in water vapour, with respect to the VSMOW. Altitudes are defined with respect to the Martian ellipsoid. fractionation factor between the ice and vapour phases during condensation and evaporation processes for the $\mathrm{D} / \mathrm{H}$ and ${ }^{18} \mathrm{O} /{ }^{16} \mathrm{O}$ ratios, respectively; these works, together with the assumption of isotopic exchange given by Rayleigh distillation, suggest that the magnitude of the variation in the ${ }^{18} \mathrm{O} /{ }^{16} \mathrm{O}$ ratio should be approximately a factor of 10 smaller than the magnitude of the variations in the $\mathrm{D} / \mathrm{H}$ ratio, depending on the temperature at which condensation occurs and the fraction of condensed water.

The lack of observed vertical variations in the isotope ratios (see Fig. 6) does not necessarily imply that fractionation during condensation is not occurring. In the wavelength range covered by ACS MIR secondary grating position 5, there is not any 
spectral signature of water ice, and dust and water ice particles cannot be distinguished. It is possible to calculate the saturation ratio, as we are sensitive to the temperature and partial pressure of $\mathrm{H}_{2} \mathrm{O}$, but the degree at which condensation is occurring is uncertain, and so is the expected oxygen isotopic fractionation, given their relation. In any case, given the retrieved uncertainty from a single MIR profile, it appears to be difficult to measure the oxygen fractionation in water ice clouds, expecting a depletion of $\delta^{18} \mathrm{O}$ of approximately $-100 \%$, depending on the temperature and the fraction of condensed water. Such fractionation will be searched by looking at overall trends of the whole ACS dataset (e.g. comparing the overall trend of isotope ratios with saturation ratio).

Simultaneous profiles of the $\mathrm{D} / \mathrm{H}$ ratio and the $\mathrm{O}$ ratios could enable a deeper understanding of the isotopic fractionation of water vapour. However, the spectral signatures of $\mathrm{HD}^{16} \mathrm{O}$ in this secondary grating position are weak. The $\mathrm{D} / \mathrm{H}$ ratio can be determined from other ACS MIR secondary grating positions and from NOMAD, which is a spectrometer on board ExoMars TGO (Vandaele et al. 2018). However, the fields of view of NOMAD and ACS MIR do not coincide, and observations must be performed using either NOMAD or ACS pointings.

Averaging of the isotope-ratio vertical profiles shows an enrichment of the heavier isotopes of $\delta^{18} \mathrm{O}=200 \pm 80 \%$ and $\delta^{17} \mathrm{O}=230 \pm 110 \%$. The derived enrichment in the ${ }^{18} \mathrm{O} /{ }^{16} \mathrm{O}$ ratio may be compared with the measurement by Webster et al. (2013) $\left(\delta^{18} \mathrm{O}=84 \pm 10 \%\right)$. Nevertheless, while ACS MIR is most sensitive usually above $10-15 \mathrm{~km}$, depending on the aerosol content in the atmosphere, Webster et al. (2013) measured the isotope ratio at the surface.

Both measured isotope ratios appear to show a similar enrichment with respect to the Earth (see Figs. 6 and 7). Massdependent fractionation predicts enrichment or depletion of ${ }^{17} \mathrm{O}$ to be approximately half of that ${ }^{18} \mathrm{O}$, which is nominally not consistent with our measurements. However, given the larger uncertainties derived for the ${ }^{17} \mathrm{O} /{ }^{16} \mathrm{O}$ ratio, mass-dependent fractionation must not be ruled out.

Enrichment of the heavier isotopes with respect to the lighter one, as compared to Earth, might be indicative of atmospheric loss throughout the Martian history. The escape of oxygen atoms is thought to be dominated by the dissociative recombination of $\mathrm{O}_{2}^{+}$(e.g. Jakosky et al. 2018). Fox \& Hać (2010) modelled the isotope effect inherent in this mechanism, deriving a ratio of the escape probability of ${ }^{18} \mathrm{O}$ to that of ${ }^{16} \mathrm{O}$ of approximately 0.4 , and thus producing an enrichment of the atmosphere in heavier isotopes. Nevertheless, it must be noted that the total enrichment is also subject to other processes, such as the isotopic composition of $\mathrm{O}_{2}^{+}$. Other processes contributing to the atmospheric escape of oxygen atoms include loss by pickup ions or sputtering of neutral atoms, which might also fractionate the oxygen isotopes (Chassefière \& Leblanc 2004).

One source of systematic uncertainty might arise from the simultaneous derivation of the parameters defining the instrument line shape and the atmospheric profiles. However, the retrievals are performed independently in several spectral windows, providing similar atmospheric profiles. The calculation of the uncertainties using the dispersion of the retrieved profiles from several spectral windows is believed to provide a realistic estimation of the true uncertainty of the retrieval, including the systematic uncertainties. In addition, the good agreement found between the ACS NIR and ACS MIR temperature measurements increases the confidence in our analysis (see Fig. 5).

The large proposed spatial and temporal coverage of the ACS MIR solar occultation observations will allow the measurement of the oxygen isotope ratios in different locations and seasons, allowing a more in-depth analysis and a search for seasonal or spatial fractionation. In addition, the presence of absorption by ${ }^{12} \mathrm{C}^{16} \mathrm{O}_{2}$ and ${ }^{18} \mathrm{O}^{12} \mathrm{C}^{16} \mathrm{O}$ in this same observational setup allows the simultaneous measurement of the ${ }^{18} \mathrm{O} /{ }^{16} \mathrm{O}$ ratio in both $\mathrm{H}_{2} \mathrm{O}$ and $\mathrm{CO}_{2}$, which might depict mixing of the isotopologues of both species. We will explore both areas of study in future papers.

Acknowledgements. The authors acknowledge funding from the UK Space Agency under grants ST/R001502/1 and ST/P001572/1. The ACS experiment is led by IKI, Space Research Institute in Moscow, assisted by LATMOS in France. The project acknowledges funding by Roscosmos and CNES. The science operations of ACS are funded by Roscosmos and ESA. A.T., O.K., A.A.F., and D.A.B. acknowledge funding under grant number 14.W03.31.0017 of the Russian government. We are grateful to all ESA ESOC, ESAC and IKI Science Operations Center personnel, whose efforts made the success of TGO possible. The research materials supporting this publication can be accessed by contacting the corresponding author. The datasets generated by the ACS instrument and analysed in this study will be available in the ESA PSA repository, https://archives.esac.esa.int/psa, after the proprietary period.

\section{References}

Aoki, S., Nakagawa, H., Sagawa, H., et al. 2015, Icarus, 260, 7

Bjoraker, G., Mumma, M., \& Larson, H. P. 1989, BAAS, 21, 991

Brown, L., Humphrey, C., \& Gamache, R. 2007, J. Mol. Spectr., 246, 1

Chassefière, E., \& Leblanc, F. 2004, Planet. Space Sci., 52, 1039

Devi, V. M., Benner, D. C., Sung, K., et al. 2017, J. Quant. Spectr. Rad. Transf., 203, 158

Encrenaz, T., DeWitt, C., Richter, M. J., et al. 2016, A\&A, 586, A62

Fedorova, A. A., Montmessin, F., Korablev, O., et al. 2019, Science, submitted

Forget, F., Hourdin, F., Fournier, R., et al. 1999, J. Geophys. Res., 104, 24155

Forget, F., Montmessin, F., Bertaux, J.-L., et al. 2009, J. Geophys. Res., 114, E01004

Fox, J. L., \& Hać, A. 2010, Icarus, 208, 176

Gamache, R. R., Farese, M., \& Renaud, C. L. 2016, J. Mol. Spectr., 326, 144

Gordon, I. E., Rothman, L. S., Hill, C., et al. 2017, J. Quant. Spectr. Rad. Transf., 203,3

Hase, F., Wallace, L., McLeod, S. D., Harrison, J. J., \& Bernath, P. F. 2010, J. Quant. Spectr. Rad. Transf., 111, 521

Hu, R. 2019, Earth Planet. Sci. Lett., 519, 192

Irwin, P., Teanby, N., de Kok, R., et al. 2008, J. Quant. Spectr. Rad. Transf., 109, 1136

Irwin, P. G. J., Toledo, D., Garland, R., et al. 2019, Icarus, 321, 550

Jakosky, B. M. 1997, Adv. Space Res., 19, 1289

Jakosky, B. M., \& Phillips, R. J. 2001, Nature, 412, 237

Jakosky, B. M., Brain, D., Chaffin, M., et al. 2018, Icarus, 315, 146

Korablev, O., Montmessin, F., Trokhimovskiy, A., et al. 2017, Space Sci. Rev., 214, 7

Krasnopolsky, V. A. 2015, Icarus, 257, 377

Krasnopolsky, V., Mumma, M., Bjoraker, G., \& Jennings, D. 1996, Icarus, 124, 553

Krasnopolsky, V. A., Maillard, J. P., Owen, T. C., Toth, R. A., \& Smith, M. D. 2007, Icarus, 192, 396

Majoube, M. 1970, Nature, 226, 1242

Merlivat, L., \& Nief, G. 1967, Tellus, 19, 122

Montmessin, F., Fouchet, T., \& Forget, F. 2005, J. Geophys. Res. E, 110, E03006

Montmessin, F., Bertaux, J.-L., Quémerais, E., et al. 2006, Icarus, 183, 403

Nier, A. O., \& McElroy, M. B. 1977, J. Geophys. Res., 82, 4341

Niles, P. B., Boynton, W. V., Hoffman, J. H., Ming, D. W., \& Hamara, D. 2010, Science, 329, 1334

Olsen, K. S., Boone, C. D., Toon, G. C., et al. 2019, J. Quant. Spectr. Rad. Transf., 236, 106590

Owen, T., Maillard, J. P., de Bergh, C., \& Lutz, B. L. 1988, Science, 240, 1767 LP

Quémerais, E., Bertaux, J.-L., Korablev, O., et al. 2006, J. Geophys. Res., 111, E09S04

Rodgers, C. D. 2000, Series on Atmospheric, Oceanic and Planetary Physics, (Singapore: World Scientific), 2

Schrey, U., Rothermel, H., Drapatz, S., \& Kaeufl, H. U. 1986, A\&A, 155, 200

Vandaele, A. C., Lopez-Moreno, J.-J., Patel, M. R., et al. 2018, Space Sci. Rev., 214,80

Vandaele, A. C., Korablev, O., Daerden, F., et al. 2019, Nature, 568, 521

Villanueva, G. L., Mumma, M. J., Novak, R. E., et al. 2015, Science, 348, 218

Webster, C. R., Mahaffy, P. R., Flesch, G. J., et al. 2013, Science, 341, 260 